\title{
Loss compensation of surface plasmon polaritons in organic/metal nanowire heterostructures toward photonic logic processing
}

\author{
Yuanchao $\mathrm{Lv}^{1,2}$, Fa Feng $\mathrm{Xu}^{1}$, Kang Wang ${ }^{1}$, Yong Jun $\mathrm{Li}^{1}$ and Yong Sheng Zhao ${ }^{1,3^{*}}$
}

\begin{abstract}
Surface plasmon polaritons (SPPs) are crucial for the development of next generation information and communication technologies. However, the ohmic losses inherent to all plasmonic devices seriously limit their practical application in on-chip photonic communications. Here, loss compensation of SPPs and their application in photonic logic processing was demonstrated in rationally designed organic/ silver nanowire heterostructures. The heterostructures were synthesized by inserting silver nanowires (AgNWs) into crystalline organic microwires, which served as a microscale optical gain medium. These heterostructures with large organic/metal interfacial areas ensured the efficient energy transfer from excitons to SPPs. Gain for subwavelength SPPs in the heterostructure was achieved through stimulated emission of strongly confined SPPs. Furthermore, cascade gain was performed to realize basic nanoscale photonic devices, such as Boolean logic units. The results would pave an alternative avenue to incorporating SPP-enhanced devices into hybrid photonic circuitry.
\end{abstract}

Keywords: surface plasmon, nanowire heterostructure, organic nanowire, loss compensation

\section{INTRODUCTION}

Surface plasmon polaritons (SPPs), the coherent electron oscillations at metal-dielectric interfaces, can highly concentrate and channel light at subwavelength scale [14], making them ideal candidates for nanoscale optical and optoelectronic devices that hold great potential in nanophotonics [5-10], imaging $[11,12]$ and highthroughput sensing [13]. Nevertheless, the ohmic losses inherent to all plasmonic devices seriously limit their practical application in on-chip photonic communications [14-17]. Introducing optical gain into the dielectric material adjacent to the metal nanostructures has been identified as an effective means to compensate for the loss [18-21]. To date, the loss compensation of SPP has been demonstrated with various gain systems, such as lanthanide ion-doped glasses [22], quantum dot (QD)-doped films [23,24] and dye solutions [25]. However, these hybrid systems suffer from either large device sizes or sophisticated fabrication techniques [26], making them unsuitable for highly integrated photonic circuits and systems.

Organic crystals with abundant intermolecular interactions allow for numerous low-dimensional nanostructures [27,28], such as nanowires and nanobelts [29], which permit the integration with plasmonic nanostructures without the limit of strict lattice matching and sophisticated epitaxial technology [30]. Especially, the embedded organic/metal hybrid systems can not only ensure the output of strongly confined SPPs at subwavelength scale [31], but also benefit the exciton-plasmon coupling for SPP compensation due to their large organic/metal interfacial areas [32,33]. More importantly, organic assemblies exhibit abundant excited-state processes and high stimulated emission cross section, making it possible to provide excellent optical gain for loss compensation of subwavelength SPPs [34]. The compensation accompanied with the nonlinear increase of SPP signals would offer an effective approach to realize imperative photonic devices, such as nanoscale logic

\footnotetext{
${ }^{1}$ Key Laboratory of Photochemistry, Institute of Chemistry, Chinese Academy of Sciences, Beijing 100190, China

${ }^{2}$ Fujian Provincial Key Laboratory of Polymer Materials, College of Chemistry and Materials Science, Fujian Normal University, Fuzhou 350007, China

${ }^{3}$ University of Chinese Academy of Sciences, Beijing 100049, China

* Corresponding author (email: yszhao@iccas.ac.cn)
} 
elements $[18,19,35]$.

Herein, we propose a strategy to realize loss compensation of SPP signals in organic/silver nanowire (AgNW) heterostructures and their application in photonic logic processing. The heterostructures were constructed by inserting AgNWs into crystalline organic microwires, which served as an effective optical gain medium at microscale. These heterostructures with large organic/metal interfacial areas permit the efficient energy transfer from excitons to SPPs. The loss compensation of SPPs was demonstrated in the heterostructures based on the stimulated emission of strongly confined SPPs. Furthermore, cascade gain was performed in an individual heterostructure with two excitation spots, which enabled us to achieve basic nanoscale Boolean logic unit. These results offer a novel understanding of the assembly mechanism of hybrid materials, and provide a valuable guidance to incorporating SPP-enhanced components into nanoscale hybrid photonic circuitry.

\section{EXPERIMENTAL SECTION}

\section{Materials}

1-(4-Bromo-2-hydroxy-phenyl)-3-[4-(1-ethyl-propyl)phenyl]-propenone (BHEP) was designed and synthesized through the classical Claisen-Schmidt condensation reaction [36]. The AgNWs were synthesized by reducing $\mathrm{AgNO}_{3}$ with ethylene glycol (EG) in the presence of polyvinylpyrrolidone (PVP). Silver nitrate $\left(\mathrm{AgNO}_{3}\right)$, PVP and EG were purchased from Aldrich Chemical Co., and used without further purification.

\section{Preparation of BHEP/AgNW heterostructures}

The AgNW solution $\left(20 \mu \mathrm{L}, 2 \mathrm{mg} \mathrm{mL}^{-1}\right)$ in ethanol was dropped on a quartz plate. After the complete volatilization of ethanol, $400 \mu \mathrm{L}$ of BHEP solution $\left(1 \mathrm{mg} \mathrm{mL}^{-1}\right)$ in cyclohexane was subsequently dropped on the same plate. With the gradual evaporation of the solvent, BHEP molecules nucleate and self-assembled into one-dimensional (1D) microwires, and thus grew around the AgNWs. As a result, the BHEP/AgNW heterostructures were obtained on the quartz plate after the complete volatilization of cyclohexane. It is worth noting that the yield of the organic/Ag heterostructure with two excitation spots is lower than that of the sample with only one excitation spot.

\section{Characterization}

The morphology of the BHEP/AgNW heterostructure was examined with scanning electron microscopy (SEM,
FEI Nova NanoSEM450) and transmission electron microscopy (TEM, JEOL JEM-2010). Photoluminescence (PL) lifetime images were taken with fluorescence-lifetime imaging microscopy (FLIM) by scanning the samples with a $405 \mathrm{~nm}$ picosecond pulse laser. The FLIM (PicoQuant) was composed of picosecond pulsed diode laser (PDL800-D), fiber coupling unit (FCU II), laser scanning microscope (Olympus FV-1000), four-channel detector router (PHR 800), and photomultiplier detector assembly (PMA Series). The absorption and fluorescence spectra were measured on a UV-visible spectrometer (Perkin-Elmer Lambda 35) and a fluorescent spectrometer (Hitachi F-7000), respectively. Bright-field optical images and fluorescence microscopy images were taken from an inverted fluorescence microscope (Nikon Ti-U), by exciting the samples with a mercury lamp. The $532 \mathrm{~nm}$ CW laser as the probe laser beam was focused on the tip of AgNW to excite the SPPs. The focused pulse laser beam $(400 \mathrm{~nm}, 150 \mathrm{fs}, 1000 \mathrm{~Hz})$ was used to pump the BHEP wires through neutral density filter and half-wave plates. The spatially resolved spectra were measured with a monochrometer (Princeton Instrument Acton SP 2300i) connected with an EMCCD (Princeton Instrument ProEM 1600B).

\section{RESULTS AND DISCUSSION}

The strategy for loss compensation of SPP in an organic/ metal heterostructure is schematically illustrated in Fig. 1a. SPPs launched by a continuous wave $(\mathrm{CW})$ probe laser propagate through the organic/metal junction and scatter into the free space from the other distal end. Upon pulsed illumination, the ordered molecular aggregates around the plasmonic waveguide would permit the energy transfer from excitons to the propagating SPPs, which leads to stimulated emission of SPPs and thus the loss compensation. Here, BHEP (Fig. 1b) was designed and synthesized as the model compound for the study on loss compensation of SPPs due to the following reasons [36]. (i) Compared with the reported compounds used in organic/metal heterostructures [32], BHEP exhibits typical excited-state intramolecular proton transfer process as an efficient four energy-level structure, which would benefit the optical gain and amplification due to the guaranteed population inversion without obvious selfabsorption (Fig. S1) [34]. (ii) The $\mathrm{Br}$ and $\mathrm{O}$ atoms of BHEP have a potential coordination effect with metals and would thus benefit the growth of crystals around the plasmonic waveguide [37]. In addition, the hydrogenbond interaction $(\mathrm{O} \cdots \mathrm{H})$ facilitates the crystal growth along a specific direction to form a $1 \mathrm{D}$ nanostructure 

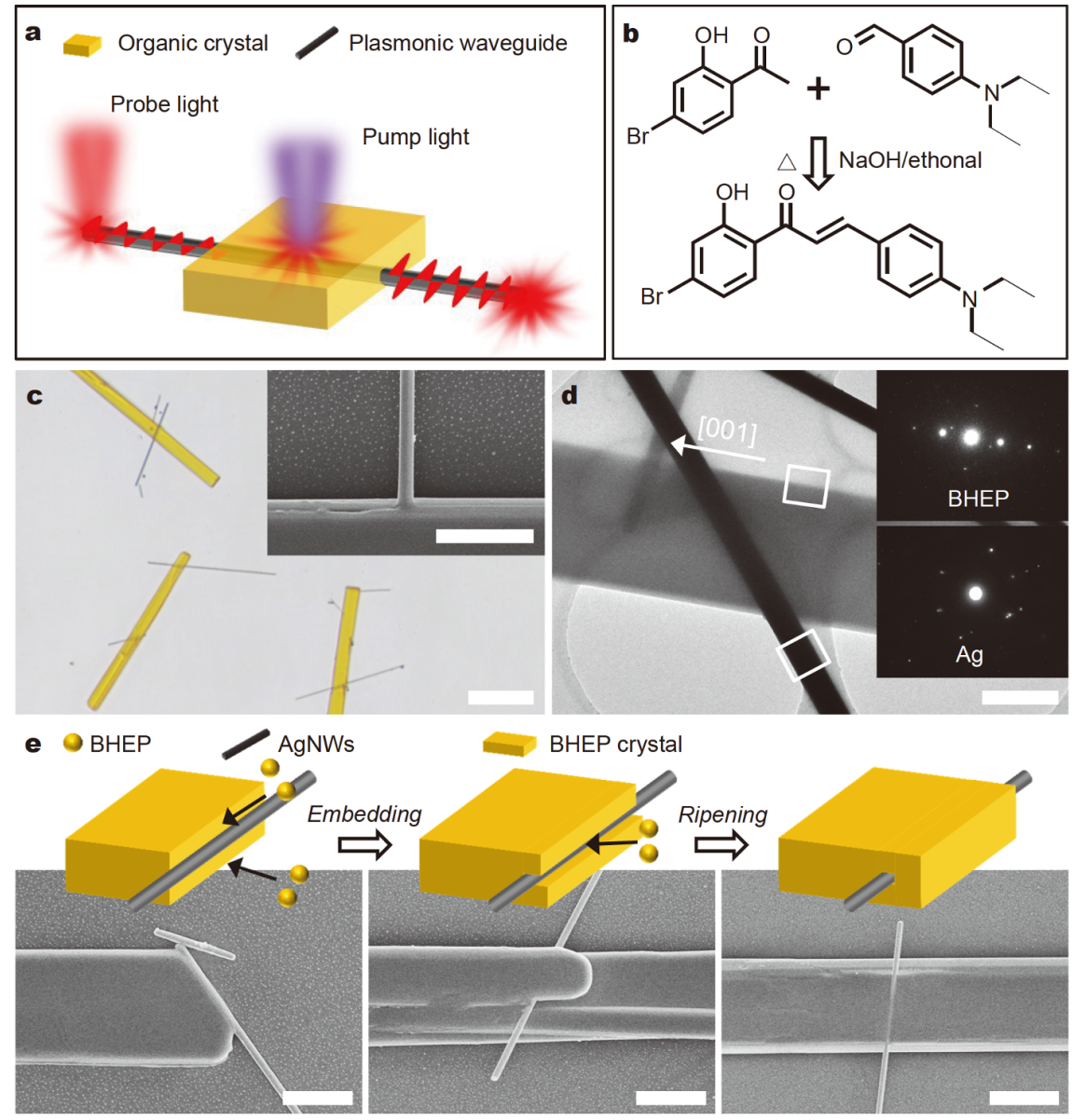

Figure 1 Design and fabrication of the BHEP/AgNW heterostructures. (a) Schematic illustration of an organic/metal heterostructure for SPP loss compensation. (b) Synthetic route of BHEP. (c) Bright-field microscopy image of the organic/metal nanowire heterostructures. Scale bar is $10 \mu \mathrm{m}$. Inset: magnified SEM image of the junction in a typical heterostructure. Scale bar is $2 \mu \mathrm{m}$. (d) TEM image of the heterostructure. Scale bar is $500 \mathrm{~nm}$. Insets: selected area electron diffraction (SAED) patterns of BHEP and AgNWs. (e) Schematic diagram for the growth process of the organic/metal nanowire heterostructures. The corresponding SEM images are also shown. Scale bars are $2 \mu \mathrm{m}$.

(Fig. S2), making it possible to achieve hybrid nanowire heterostructure with small device footprint. As for the plasmonic materials, chemically synthesized AgNWs with diameters of $\sim 180 \mathrm{~nm}$ were utilized to support the SPPs due to their atomically smooth surfaces and low propagation loss (Fig. S3). All the AgNWs used in this work were not coated with the surfactants.

The heterostructures were prepared by embedding AgNWs in BHEP crystals during crystal growth in the liquid phase (Fig. S4). In a typical preparation, AgNWs dispersed in ethanol $\left(2 \mathrm{mg} \mathrm{mL}^{-1}\right)$ were first drop-cast onto a quartz wafer, and then $0.5 \mathrm{~mL}$ of BHEP solution (cyclohexane, $1 \mathrm{mg} \mathrm{mL}^{-1}$ ) was dispersed on the same substrate. With the evaporation of the solvent, the molecules self-assembled into $1 \mathrm{D}$ microwires, and grew around the AgNWs. As a result, BHEP/AgNW hetero- structures were obtained and dispersed on the substrates for the subsequent characterizations (Fig. 1c). The SEM image (Fig. 1c, insert) indicates that the AgNWs are partially embedded in the organic microstructures, which can ensure a stable exciton-SPP coupling insensitive to mechanical vibrations $[30,38]$. The adopted diameter of AgNWs is $\sim 180 \mathrm{~nm}$, and the corresponding field energy portion of the plasmon mode on the surface is nearly $80 \%$ [39], which is suitable for coupling with the gain molecules nearby. Meanwhile, the thickness of the molecular layer is supposed to be slightly higher than the AgNW diameters, which would make AgNWs surrounded by abundant gain molecules and thus benefit the SPP loss compensation. The organic single-crystalline nanowire at the junction (Fig. 1d) possesses low scattering density and smooth surfaces, thus making it possible to provide effi- 
cient optical gain for loss compensation of subwavelength SPP signals.

To investigate the growth mechanism of the heterostructures, we studied the evolution of their morphology over growth time (Fig. 1e). At the initial stage, AgNWs were dispersed in the solvent due to the wetting of the solvent that can reduce the van der Waals interaction between the AgNWs and the substrate. During the evaporation of the solvent, the BHEP molecules nucleated and assembled into 1D crystals, and then the AgNWs were stuck on the organic microwires. The BHEP crystals nearby the AgNWs grew around the wire driven by avoiding the major disruption of lattice structure and reducing the overall interfacial energy, which result in the final embedded heterostructures. Following this growth process, we can adjust the width of the nanowire to modulate the gain region by changing the concentration of BHEP units. For instance, the width of nanowires can be tuned from $\sim 2$ to $8 \mu \mathrm{m}$ by changing the concentration of BHEP molecules from 1 to $4 \mathrm{mg} \mathrm{mL}^{-1}$ (Fig. S5).

These embedded heterostructures provide much larger organic/metal interfacial area as compared with the junction constructed by simple point contact, which would be beneficial for the energy transfer from excitons to SPPs in the AgNWs [32,40]. This merit was demonstrated by studying the decay of excitons in a typical heterostructure as shown in Fig. 2a. Here, the decay kinetics was characterized by FLIM, which can be used to record the fluorescence lifetimes of chromophores at each spatially resolvable element of a microscope image. The distribution of the lifetime manifests that the PL decay at the junction is obviously faster than that at the body of the organic wire (Fig. 2b, c and Fig. S6). The faster decay reveals that the larger contact area of the embedded structure would benefit the energy transfer from excitons to the SPPs. Under UV (330-380 nm) excitation, small bright red spots were observed at the AgNW distal ends (Fig. S7), which not only exhibit the typical feature of the SPP waveguide, but also confirm the efficient excitonplasmon coupling at the embedded sections.

In the crystalline heterostructure, the highly ordered molecular aggregates would induce the gain anisotropy around AgNWs [32], facilitating the investigtion of the relationship between the pump polarization and excitation efficiency for the realization of high optical gain. Here, a single heterostructure was excited with a linearly polarized pump laser (400 nm, $150 \mathrm{fs}$ ), and the parameter $\theta$ refers to the angle between the long axis of the BHEP
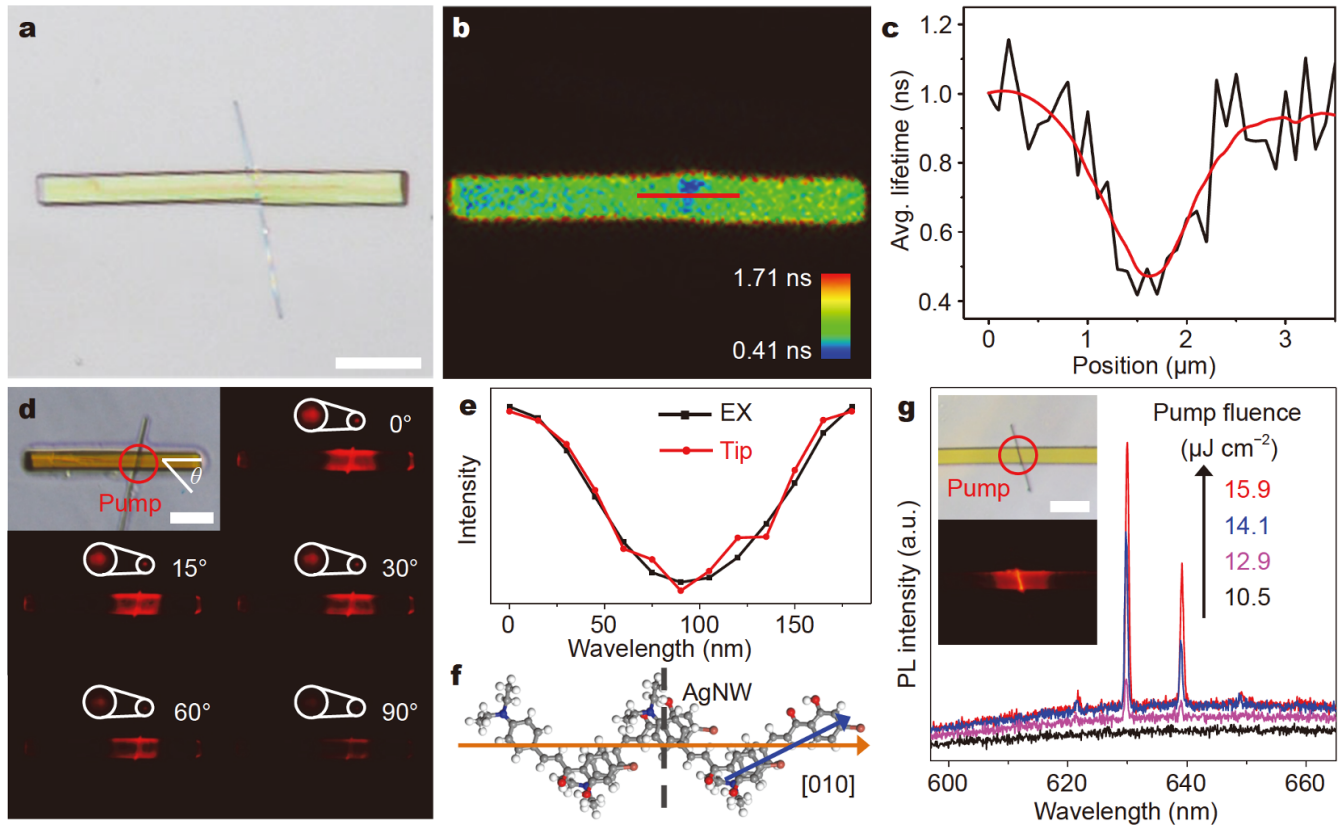

Figure 2 Optical characterizations of the BHEP/AgNW heterostructures. (a) Bright-field microscopy of an embedded nanowire heterostructure. Scale bar is $5 \mu \mathrm{m}$. (b) FLIM image of the heterostructure in (a). (c) Average lifetimes across the junction (marked with red line in (b)). (d) Bright-field and PL images of the heterostructures with different cross angles between the pump polarizations and long axis of the BHEP microwire. Scale bar is $5 \mu \mathrm{m}$. (e) Normalized PL intensities $I_{\theta}$ versus $\theta$. $I_{\theta}$ is the SPP intensity detected at the BHEP/Ag junction. (f) The spatial relationship among BHEP molecular transition dipole moment (blue arrow) and the preferential grow direction (orange arrow) of the BPEA NW. (g) PL spectra collected from the junction with the pump laser polarization parallel to the BHEP wire. Inset: Bright-field and PL images of the heterostructure. Scale bar is $5 \mu \mathrm{m}$. 
wire and the pump laser polarization (Fig. 2d). It was observed that when $\theta$ was $0^{\circ}-30^{\circ}$, the emission from the exciting point of BHEP NW was much stronger than that when $\theta$ was increased to $60^{\circ}-90^{\circ}$, which reveals that the pump polarizations would significantly influence the optical gain in the hybrid system. The corresponding emission intensity oscillates between the maximum and the minimum values with $\theta$ ranging in a period of $180^{\circ}$ (Fig. 2e), which should be a direct consequence of the polarization-dependent excitation efficiency. As shown in Fig. $2 \mathrm{f}$, the BHEP molecules stack nearly parallel to the $b$ axis, tilted at an angle of $26^{\circ}$, and the transition dipole of BHEP is along the molecular long axis (Fig. S8). Therefore, when $\theta$ is $0^{\circ}$ or $180^{\circ}$, namely, the pump laser polarization is parallel to the BHEP wire, the largest excitation efficiency can be obtained. As shown in Fig. 2g, with increasing pump pulse energy over a threshold (Fig. S9), sharp PL peaks in the gain region was observed and amplified, which indicated the lasing action in the heterostructure, showing great potential to offer the ef- ficient optical gain for SPP loss compensation.

These heterostructures with efficient exciton-plasmon coupling and high optical gain provide an opportunity to investigate the loss compensation of SPPs. The gain measurements were carried out on a home-built far-field microphotoluminescence system (Fig. S10). As shown in Fig. 3a, SPPs were launched by focusing a $635 \mathrm{~nm} \mathrm{CW}$ probe laser beam at the left tip of AgNW, and was supposed to be loss-compensated through stimulated emission by focusing the linearly polarized pump laser on the BHEP-AgNW junction [25]. SPP gain would be achieved if the output signal at the right NW tip in panel III (pump + probe) was enhanced compared with the value obtained from panel I (probe only) after correcting for the background spontaneous emission in panel II (pump only). As shown in Fig. 3b, a clear enhancement of the output signal was observed with optical pumping, i.e., $I_{\text {pump+probe }}$ $-I_{\text {pump }}>I_{\text {probe. }}$ To evaluate the internal optical gain, the output signal enhancement factor was defined as: $f=$ $\left(I_{\text {pump+probe }}-I_{\text {pump }}\right) / I_{\text {probe }}$, where $I$ refers to the peak value
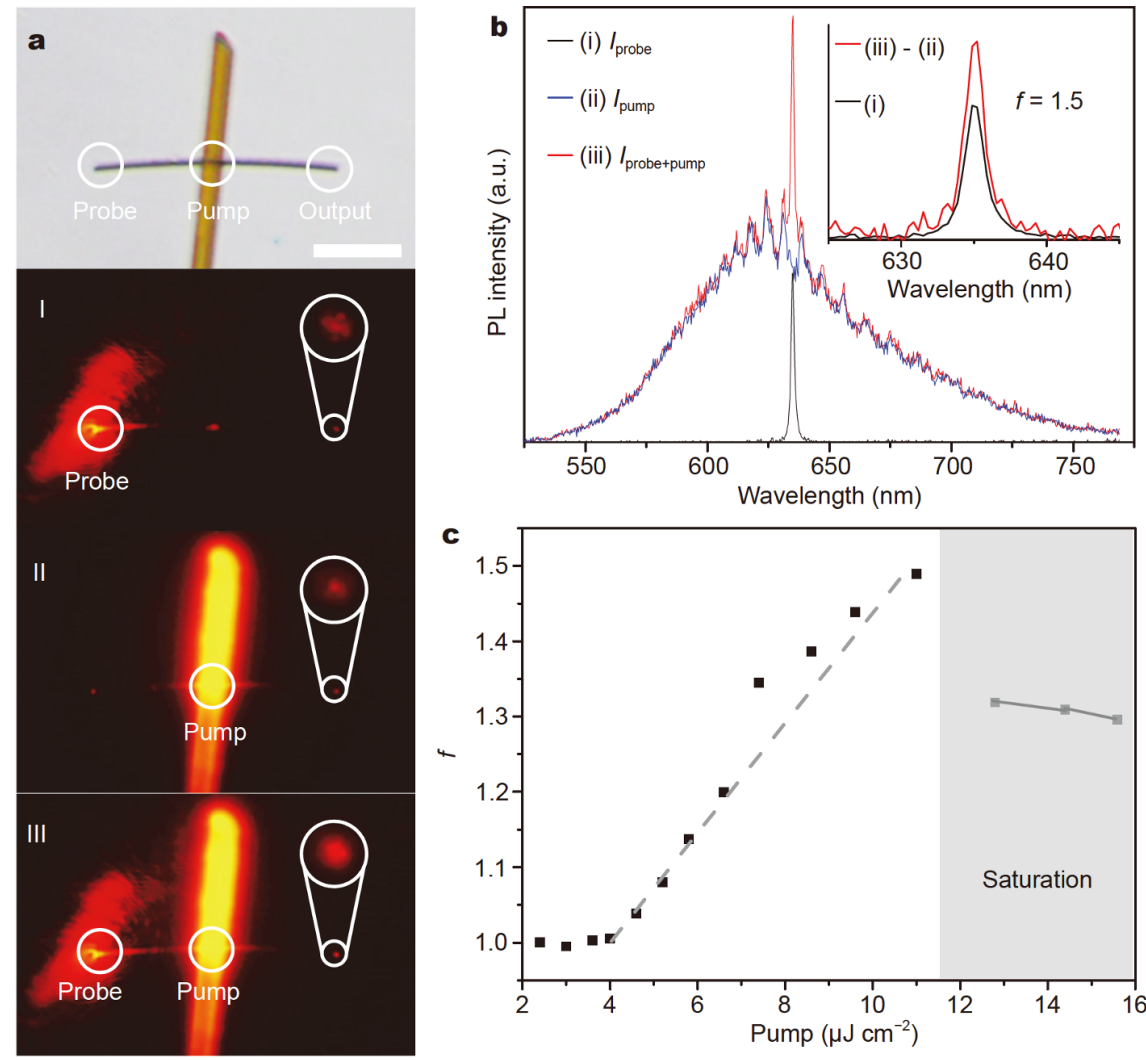

Figure 3 Loss compensation of SPPs. (a) Bright-field and PL images of the heterostructure excited by a probe laser (I), a pump laser (II), and both the probe and pump lasers (III), respectively. Scale bar is $5 \mu \mathrm{m}$. (b) PL spectra collected from the right tip of the AgNWs under the three different excitation conditions shown in (a): (i) probe only; (ii) pump only; (iii) pump + probe. The inset shows that an output signal enhancement factor of $f=$ 1.5 was obtained from the spectra at the probe wavelength. (c) Plots of SPP signal enhancement factor $f$ vs. the pump intensity. 
of the corresponding intensity at $635 \mathrm{~nm}$ [33]. The inset in Fig. $3 \mathrm{~b}$ demonstrates a 1.5 -fold enhancement of the SPP output at a pump power of $11.2 \mu \mathrm{J} \mathrm{cm}^{-2}$.

A clear threshold for SPP gain was observed as a function of pump irradiance (Fig. 3c), while maintaining the constant power of the probe laser and the polarization of the probe laser. The SPP signal enhancement factor of $f>1$ was evident at a pump irradiance of $\sim 4 \mu \mathrm{J} \mathrm{cm}^{-2}$, and $f$ increased linearly with pump irradiance above this threshold. The observation of both threshold and linear pump power dependence are signatures of stimulated SPP emission [18,41]. For larger pump powers $\left(>12 \mu \mathrm{J} \mathrm{cm}^{-2}\right)$, lasing in the organic crystal was observed and became the dominant gain process, which suppressed the further enhancement of SPP signals (Fig. S11). We can speculate that the SPP-enhanced process as well as the performances of nanophotonic devices could be improved by properly designing the hybrid structure $[42,43]$.

Thanks to the flexibility of the liquid-phase fabrication method, self-assembled organic/Ag heterostructures permit the AgNW to be embedded into more than one organic wire [44], which may offer cascade gain process, and prompt us to perform optical signal processing at the nanoscale [45]. For example, the AgNW was simultaneously embedded into two organic wires (Fig. 4a), resulting in two individual heterojunctions in a single system. The pulse laser was split into two beams to pump the two BHEP-AgNW junctions, respectively (Fig. S10). The corresponding PL microscopy images in Fig. 4b exhibit the four different combinations of two pump lasers as input signals. In this heterostructure, the excitation spot with optical pumping was defined as 1 , while that without optical pumping was defined as 0 . Then these four images represent four combinations of input values: $(1,1),(1,0),(0,1)$ and $(0,0)$, respectively, which is in accordance with the Boolean logic inputs. Such heterojunction forms a nanoscale logic gate with two input terminal (I1 and I2) and one output terminal (O). The value of the SPP output was obtained after correcting for the background spontaneous emission.

The function of the gate depends on the intensities of SPPs scattered at O port for different gain cases (Fig. 4c). Assuming that the maximum in Fig. $4 \mathrm{~d}$ is 1 arbitrary unit (au), we find that $\mathrm{O}(1,1)=1 \mathrm{au}$, $\mathrm{O}$
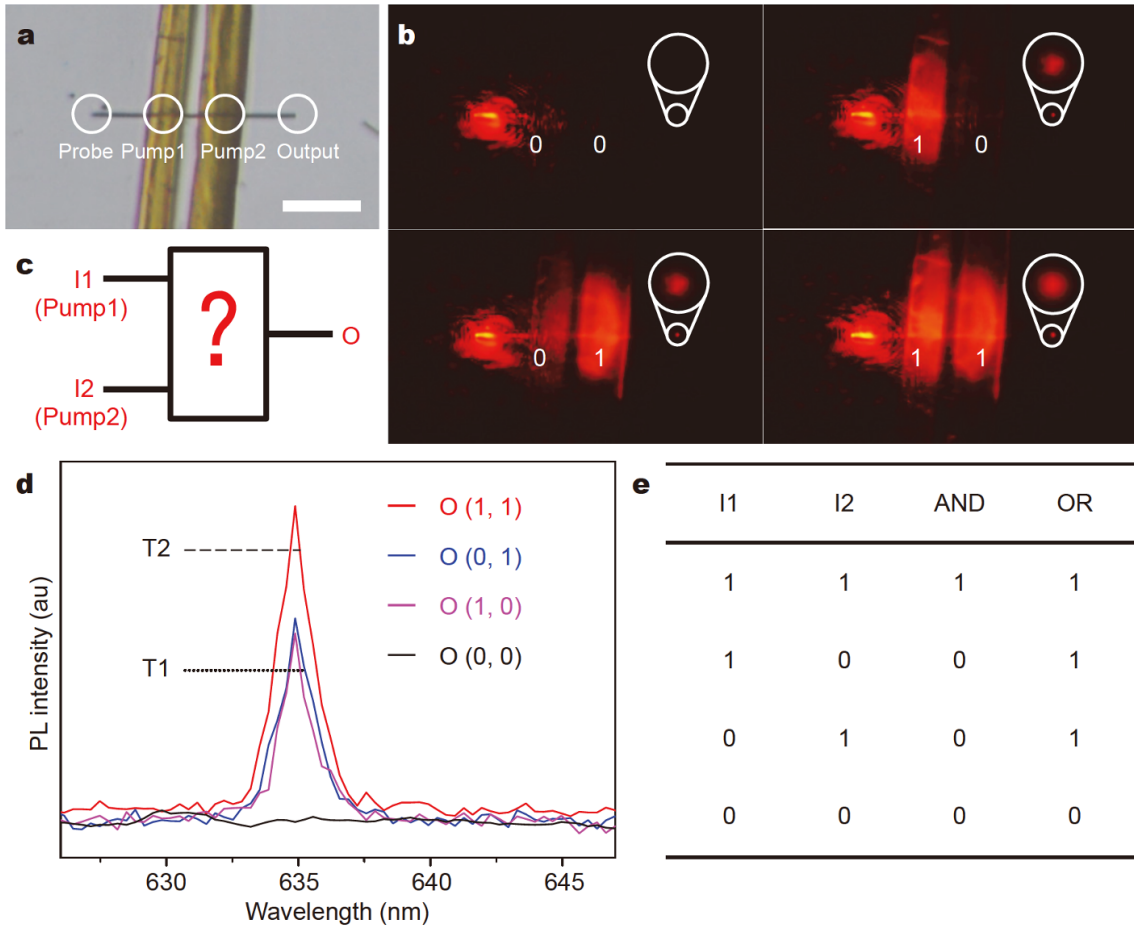

Figure 4 Photonic logic processing based on loss compensation of SPPs. (a) Bright-field image of the heterostructure with two excitation spots. Scale bar is $5 \mu \mathrm{m}$. (b) PL microscopy images under the excitations of four different combinations of the two excitation spots. Here, 1 and 0 were defined respectively according to the optical gain in the junction. (c) The formed basic logic gate with two simultaneous inputs and one output signal. (d) The scattering spectra of SPPs at the O terminal under the excitations shown in (b) after correcting for the background spontaneous emission. T1 and T2 represent the thresholds for OR and AND gates, respectively. (e) Summary of optical logic operations for AND and OR gates. 
$(0,1)=\mathrm{O}(1,0)=0.55 \mathrm{au}$, and $\mathrm{O}(0,0)=0 \mathrm{au}$. By defining an intensity threshold (T) to separate $\mathrm{ON}$ and OFF states among these values, either OR or AND logic operations could be achieved in such hybrid structure. For example, for a threshold of $0.4 \mathrm{au}(\mathrm{T} 1$, the dotted line in Fig. $4 \mathrm{~d}),(\mathrm{I} 1=1, \mathrm{I} 2=1)$ results in $\mathrm{O}=1,(\mathrm{I} 1=1, \mathrm{I} 2=0)$ results in $\mathrm{O}=1$, and $(\mathrm{I} 1=0, \mathrm{I} 2=1)$ results in $\mathrm{O}=1$, showing the performance of an OR gate. While, when the higher threshold (such as T2 $=0.85 \mathrm{au}$, the dashed line in Fig. 4 d) was defined, only $(\mathrm{I} 1=1, \mathrm{I} 2=1)$ results in $\mathrm{O}=1$, and all other three input combinations lead to $\mathrm{O}=0$, which serves as an AND gate. The total outputs with different thresholds have been summarized in Fig. 4e, demonstrating an OR or AND gate. We believe that this concept can be further generalized and expanded to more complex SPP-enhanced components to serve as various nanoscale devices, such as splitters, dichroic mirrors, routers, and so on [46].

\section{CONCLUSIONS}

In summary, we report a type of embedded organic/silver nanowire heterostructure to realize loss compensation of subwavelength signals and their application in photonic logic processing. The heterostructures were constructed by inserting AgNWs into crystalline organic microwires, which functioned as a microscale optical gain medium. Due to the large organic/metal interfacial area, these heterostructures exhibited efficient exciton-plasmon coupling, and transferred the exciton energy to SPPs. Loss compensation of SPPs was demonstrated in the heterostructure based on stimulated emission of strongly confined SPPs. Furthermore, the individual heterostructure with two excitation spots possessed cascade gain, which enabled us to achieve basic Boolean logic unit at nanoscale. These results offer a novel understanding of the assembly mechanism of hybrid materials, and are essential for incorporating plasmonic amplifiers as practical components into high-capacity photonic circuits.

Received 24 September 2019; accepted 7 November 2019; published online 6 December 2019

1 Lal S, Link S, Halas NJ. Nano-optics from sensing to waveguiding. Nat Photon, 2007, 1: 641-648

2 Eaton SW, Fu A, Wong AB, et al. Semiconductor nanowire lasers. Nat Rev Mater, 2016, 1: 16028

3 Oulton RF, Sorger VJ, Zentgraf T, et al. Plasmon lasers at deep subwavelength scale. Nature, 2009, 461: 629-632

4 Sidiropoulos TPH, Röder R, Geburt S, et al. Ultrafast plasmonic nanowire lasers near the surface plasmon frequency. Nat Phys, 2014, 10: 870-876

5 Noginov MA, Zhu G, Belgrave AM, et al. Demonstration of a spaser-based nanolaser. Nature, 2009, 460: 1110-1112

6 Wei H, Wang Z, Tian X, et al. Cascaded logic gates in nanophotonic plasmon networks. Nat Commun, 2011, 2: 387

7 Zhang Q, Li G, Liu X, et al. A room temperature low-threshold ultraviolet plasmonic nanolaser. Nat Commun, 2014, 5: 4953

8 Fang Z, Zhu X. Plasmonics in nanostructures. Adv Mater, 2013, 25: 3840-3856

9 Akimov AV, Mukherjee A, Yu CL, et al. Generation of single optical plasmons in metallic nanowires coupled to quantum dots. Nature, 2007, 450: 402-406

10 Nezhad MP, Simic A, Bondarenko O, et al. Room-temperature subwavelength metallo-dielectric lasers. Nat Photon, 2010, 4: 395399

11 Wang D, Yang A, Wang W, et al. Band-edge engineering for controlled multi-modal nanolasing in plasmonic superlattices. Nat Nanotech, 2017, 12: 889-894

12 Tseng ML, Yang J, Semmlinger M, et al. Two-dimensional active tuning of an aluminum plasmonic array for full-spectrum response. Nano Lett, 2017, 17: 6034-6039

13 Galanzha EI, Weingold R, Nedosekin DA, et al. Spaser as a biological probe. Nat Commun, 2017, 8: 15528

14 Liu N, Wei H, Li J, et al. Plasmonic amplification with ultra-high optical gain at room temperature. Sci Rep, 2013, 3: 1967

15 Li DB, Ning CZ. Giant modal gain, amplified surface plasmonpolariton propagation, and slowing down of energy velocity in a metal-semiconductor-metal structure. Phys Rev B, 2009, 80: 153304

16 Khurgin JB. How to deal with the loss in plasmonics and metamaterials. Nat Nanotechnol, 2015, 10: 2-6

17 Fedyanin DY, Krasavin AV, Arsenin AV, et al. Surface plasmon polariton amplification upon electrical injection in highly integrated plasmonic circuits. Nano Lett, 2012, 12: 2459-2463

18 Gather MC, Meerholz K, Danz N, et al. Net optical gain in a plasmonic waveguide embedded in a fluorescent polymer. Nat Photon, 2010, 4: 457-461

19 De Leon I, Berini P. Amplification of long-range surface plasmons by a dipolar gain medium. Nat Photon, 2010, 4: 382-387

20 Wu X, Xiao Y, Meng C, et al. Hybrid photon-plasmon nanowire lasers. Nano Lett, 2013, 13: 5654-5659

21 Kéna-Cohen S, Stavrinou PN, Bradley DDC, et al. Confined surface plasmon-polariton amplifiers. Nano Lett, 2013, 13: 1323-1329

22 Ambati M, Nam SH, Ulin-Avila E, et al. Observation of stimulated emission of surface plasmon polaritons. Nano Lett, 2008, 8: 39984001

23 Grandidier J, des Francs GC, Massenot S, et al. Gain-assisted propagation in a plasmonic waveguide at telecom wavelength. Nano Lett, 2009, 9: 2935-2939

24 Kress SJP, Cui J, Rohner P, et al. A customizable class of colloidalquantum-dot spasers and plasmonic amplifiers. Sci Adv, 2017, 3 : e1700688

25 Chen YH, Li J, Ren ML, et al. Direct observation of amplified spontaneous emission of surface plasmon polaritons at metal/dielectric interfaces. Appl Phys Lett, 2011, 98: 261912

26 Yan R, Pausauskie P, Huang J, et al. Direct photonic-plasmonic coupling and routing in single nanowires. Proc Natl Acad Sci USA, 2009, 106: 21045-21050

27 Takazawa K, Inoue J, Mitsuishi K, et al. Micrometer-scale photonic circuit components based on propagation of exciton polaritons in organic dye nanofibers. Adv Mater, 2011, 23: 3659-3663

28 Fang HH, Yang J, Feng J, et al. Functional organic single crystals 
for solid-state laser applications. Laser Photonics Rev, 2014, 8: 687-715

29 Liao Q, Jin X, Zhang H, et al. An organic microlaser array based on a lateral microcavity of a single j-aggregation microbelt. Angew Chem Int Ed, 2015, 54: 7037-7041

30 Li YJ, Lv Y, Zou CL, et al. Output coupling of perovskite lasers from embedded nanoscale plasmonic waveguides. J Am Chem Soc, 2016, 138: 2122-2125

31 Pyayt AL, Wiley B, Xia Y, et al. Integration of photonic and silver nanowire plasmonic waveguides. Nat Nanotech, 2008, 3: 660-665

32 Li YJ, Hong Y, Peng Q, et al. Orientation-dependent excitonplasmon coupling in embedded organic/metal nanowire heterostructures. ACS Nano, 2017, 11: 10106-10112

33 Paul A, Zhen YR, Wang Y, et al. Dye-assisted gain of strongly confined surface plasmon polaritons in silver nanowires. Nano Lett, 2014, 14: 3628-3633

34 Park S, Kwon OH, Kim S, et al. Imidazole-based excited-state intramolecular proton-transfer materials: Synthesis and amplified spontaneous emission from a large single crystal. J Am Chem Soc, 2005, 127: 10070-10074

35 Wang F, Gong Z, Hu X, et al. Nanoscale on-chip all-optical logic parity checker in integrated plasmonic circuits in optical communication range. Sci Rep, 2016, 6: 24433

36 Cheng X, Wang K, Huang S, et al. Organic crystals with nearinfrared amplified spontaneous emissions based on $2^{\prime}$-hydroxychalcone derivatives: Subtle structure modification but great property change. Angew Chem, 2015, 127: 8489-8493

37 Henary MM, Fahrni CJ. Excited state intramolecular proton transfer and metal ion complexation of 2-(2'-hydroxyphenyl)benzazoles in aqueous solution. J Phys Chem A, 2002, 106: 5210-5220

38 Lv Y, Li YJ, Li J, et al. All-color subwavelength output of organic flexible microlasers. J Am Chem Soc, 2017, 139: 11329-11332

39 Zou CL, Sun FW, Xiao YF, et al. Plasmon modes of silver nanowire on a silica substrate. Appl Phys Lett, 2010, 97: 183102

$40 \mathrm{Zu} \mathrm{S}$, Li B, Gong Y, et al. Active control of plasmon-exciton coupling in $\mathrm{MoS}_{2}-\mathrm{Ag}$ hybrid nanostructures. Adv Opt Mater, 2016, 4: 1463-1469

41 Noginov MA, Zhu G, Mayy M, et al. Stimulated emission of surface plasmon polaritons. Phys Rev Lett, 2008, 101: 226806

42 Guo X, Qiu M, Bao J, et al. Direct coupling of plasmonic and photonic nanowires for hybrid nanophotonic components and circuits. Nano Lett, 2009, 9: 4515-4519

43 Cho $\mathrm{CH}$, Aspetti CO, Turk ME, et al. Tailoring hot-exciton emission and lifetimes in semiconducting nanowires via whispering-gallery nanocavity plasmons. Nat Mater, 2011, 10: 669-675

44 Li YJ, Yan Y, Zhang C, et al. Embedded branch-like organic/metal nanowire heterostructures: Liquid-phase synthesis, efficient photon-plasmon coupling, and optical signal manipulation. Adv Mater, 2013, 25: 2784-2788

45 Yan Y, Zhang C, Zheng JY, et al. Optical modulation based on direct photon-plasmon coupling in organic/metal nanowire heterojunctions. Adv Mater, 2012, 24: 5681-5686

46 Bozhevolnyi SI, Volkov VS, Devaux E, et al. Channel plasmon subwavelength waveguide components including interferometers and ring resonators. Nature, 2006, 440: 508-511

Acknowledgements This work was supported by the Ministry of Science and Technology of China (2017YFA0204502) and the National Natural Science Foundation of China (21533013 and 21790364).
Author contributions Zhao YS conceived the idea and supervised the project. Lv Y designed the experiments and prepared the materials. Lv Y and Xu FF performed the optical measurements. Lv Y, Xu FF and Wang $\mathrm{K}$ put forward the theoretical model and contributed to the theoretical calculations. Lv Y, Li YJ and Zhao YS analyzed the data. Lv Y, Li YJ, and Zhao YS wrote the manuscript. All authors discussed the results and commented on the manuscript.

Conflict of interest The authors declare that they have no conflict of interest.

Supplementary information Supporting data are available in the online version of the paper.

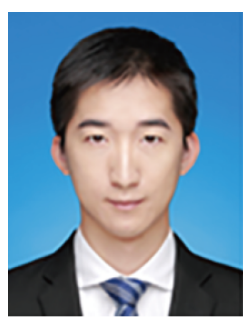

Yuanchao Lv received his $\mathrm{PhD}$ degree in physical chemistry at the Institute of Chemistry, Chinese Academy of Sciences (ICCAS) in 2018. He is currently an associate professor in the College of Chemistry and Materials Science, Fujian Normal University. His research interests are the photonic properties of porous micro/nanomaterial, organic/metal hybrid systems and their application as miniaturized photonic devices.

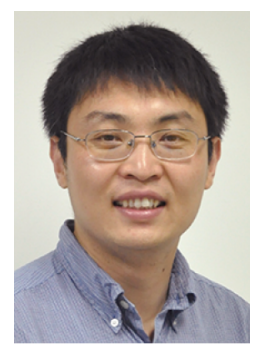

Yong Sheng Zhao received his $\mathrm{PhD}$ degree in 2006 at ICCAS. After that, he joined the University of California at Los Angeles (UCLA) and Northwestern University as a postdoctoral fellow. In 2009, he returned to ICCAS as a professor of chemistry. His research interests include the controllable synthesis of low-dimensional organic materials, photophysical and photochemical processes, as well as the fabrication and performance optimization of photonic/optoelectronic devices.

\section{有机/金属纳米线异质结中的SPPs损耗补偿用于 光学逻辑运算}

\author{
吕远超 ${ }^{1,2}$, 徐法峰 ${ }^{1}$, 王康 ${ }^{1}$, 李勇军 ${ }^{1}$, 赵永生 ${ }^{1,3^{*}}$
}

摘要 表面等离子体激元(SPPs)对于下一代信息通信技术的发展 至关重要. 然而, SPPs器件固有的欧姆损耗限制了它们在片上光学 通讯中的实际应用. 本工作中, 我们在合理设计的有机/金属纳米线 异质结中实现了 SPPs的损耗补偿及其在光学逻辑运算中的应用. 通过将银纳米线 ( $\mathrm{AgNWs}$ ) 插入结晶有机微丝中来合成异质结构, 并将其用作微米级光学增益介质. 所制备的异质结具有较大的有 机/金属界面面积, 能确保从激子到SPPs的高效能量转移. 异质结 构中亚波长SPPs的增益是通过强烈限制SPPs的受激发射实现的. 此外, 我们还实现了SPPs的级联增益, 并将其用于构建微纳光子学 器件, 例如Boolean逻辑单元. 相关结果为具有SPPs增强功能的器件 用于混合光子学回路提供了新的思路. 